\title{
The mechanism of non-linear photochemical oscillations in the mesopause region
}

\author{
M. Yu. Kulikov ${ }^{1,2}$, O. L. Vadimova ${ }^{1}$, S. K. Ignatov ${ }^{2,1}$, and A. M. Feigin ${ }^{1,2}$ \\ ${ }^{1}$ Institute of Applied Physics of the Russian Academy of Sciences, 46 Ulyanov Str., 603950, Nizhny Novgorod, Russia \\ ${ }^{2}$ Lobachevsky State University of Nizhny Novgorod, 23 Gagarin Ave., 603950, Nizhny Novgorod, Russia
}

Correspondence to: M. Yu. Kulikov (kulm@appl.sci-nnov.ru)

Received: 11 October 2011 - Revised: 16 April 2012 - Accepted: 29 July 2012 - Published: 6 September 2012

\begin{abstract}
The mechanism of generation of 2-day photochemical oscillations in the mesopause region $(80-90 \mathrm{~km})$ has been studied analytically. The initial system of equations of chemical kinetics describing the temporal evolution of $\mathrm{O}$, $\mathrm{O}_{3}, \mathrm{H}, \mathrm{OH}$ and $\mathrm{HO}_{2}$ concentrations with allowance for diurnal variations of solar radiation has been simplified successively to a system of two nonlinear first-order time equations with sinusoidal external forcing. The obtained system has a minimum number of terms needed for generation of 2day oscillations. Linearization of this system near the perioddoubling threshold permits separating explicitly a particular case of the Mathieu equation $\ddot{x}+\alpha \cdot \sin \omega t \cdot x=0$, in which the first sub-harmonic $(\omega / 2)$ of the exciting force starts to grow exponentially when the amplitude of external forcing $(\alpha)$ exceeds its threshold value. Finally, a system of two simplest differential equations with power-law nonlinearity has been derived that allows analytical investigation of the effect of arising of reaction-diffusion waves in the mesospheric photochemical system.
\end{abstract}

\section{Introduction}

An intriguing photochemical property of the mesosphere is that this system can respond nonlinearly to diurnal variations of solar radiation. Sonnemann and Fichtelmann (1987, 1997), Fichtelmann and Sonnemann (1992), Feigin et al. (1998), and Konovalov and Feigin (2000) used zerodimensional models (without spatial transport) to show that a wide spectrum of periodic (subharmonic, with periods of 2, 3, 4, and more days) and chaotic regimes of behavior of minor gas constituents may exist at the heights of the mesopause region $(80-90 \mathrm{~km})$. Note that diurnal variation of solar radiation is the key mechanism of the Sun's influence on the chemical process in the entire atmosphere of the Earth. However, no other atmospheric photochemical systems possessing similar nonlinear dynamic properties are known to date. Besides, the interest in nonlinear oscillations in the mesopause region is associated with the fact that they may influence the behavior of key characteristics of the mesosphere. The point is that exothermal reactions between the chemical constituents of the mesospheric photochemical system ensure the main photochemical heating of the mesospheric air (and the rate of the heat influx caused by these processes) reaches $1-2 \mathrm{~K}$ per day at the heights of the nonlinear-response region (Sonnemann et al., 1999). Therefore, nontrivial behavior of minor gas constituents can manifest noticeably in the variability of air temperature in the upper mesosphere and, consequently, in variations of other characteristics of this region. Specifically, the presence of the constituent with a period of $48 \mathrm{~h}$ in the spectrum of air heating variations suggests that there is a connection (Sonnemann and Fichtelmann, 1997) between nonlinear photochemical oscillations and the so-called excitation of quasi-2day waves. These powerful atmospheric waves have been observed regularly in the mesosphere and lower thermosphere for more than $40 \mathrm{yr}$ already (starting with Doyle, 1968 and Muller, 1972), but possible mechanisms of their excitation as a function of latitude and season are still being discussed (see, e.g. Sandford et al., 2008).

Further studies of the nonlinear response of mesospheric photochemistry (Sonnemann et al., 1999; Sonnemann and Feigin, 1999; Sonnemann, 2001; Kulikov and Feigin, 2004, 2005; Kulikov, 2004, 2007; Sonnemann and Grygalashvyly, 2005; Kulikov and Gashturi, 2006) revealed that behavior of the concentrations of minor gas constituents may be simpler 
or more complicated, depending on atmospheric transport (eddy diffusion, winds in the vertical and horizontal directions, or quasi-2-day atmospheric waves). For example, Kulikov and Feigin $(2004,2005)$ found that the influence of the horizontal eddy diffusion leads to the formation of a new class of nonlinear reaction-diffusion waves in the form of moving fronts of the oscillating phases of minor gas constituents traveling with constant velocity in the zonal direction. Specifically, it was shown that for the oscillations with a period of $48 \mathrm{~h}$, the propagation velocity of such waves is directly proportional to the coefficient of the horizontal eddy diffusion $(D)$ and reaches $100 \mathrm{~km}$ per day for the coefficients typical of that diffusion (Ebel, 1980). Therefore, potential experimental registration of reaction-diffusion waves of minor gas constituents in the upper mesosphere (using satellitebased methods or rocket-borne measurements) allows determining the coefficient of horizontal eddy diffusion that has not so far been measured in the upper mesosphere.

The above mentioned studies were performed mainly by numerical simulations. No simple physical model of the mechanism of formation of the nonlinear response of the mesospheric photochemical system has been developed up to now. Meanwhile, without knowledge of this mechanism it is difficult to reveal possible causes of the mentioned effects in the photochemistry of the stratosphere and troposphere. Besides, reaction-diffusion waves have not been studied. To all appearances, the mechanism of generation of these waves markedly differs from that of classical waves arising, for instance, in bistable chemical systems where the concentration front speed is proportional to $\sqrt{D}$.

In this paper we study analytically the mechanism of generation of 2-day photochemical oscillations in the mesopause region. To do so, we simplify the initial system of equations successively (retaining the qualitative properties, rather than seeking the quantitative correspondence) and reduce it to a system of two simplest, yet still nonlinear first-order time equations with sinusoidal external forcing with a period of $24 \mathrm{~h}$. Linearization of this system near the threshold of period doubling allows us to single out explicitly a particular case of the Mathieu equation in which an instability of the first subharmonic of this forcing is known to arise at a sufficiently high amplitude of external forcing. We expand the initial system of equations and formulate a simplified system of two differential nonlinear equations which fits best the study of the effect of formation of reaction-diffusion waves in the mesospheric photochemical system. It is shown that, similarly to the initial system, the obtained system of equations has subharmonic oscillations with a period of $48 \mathrm{~h}$, as well as, generally speaking, a wide spectrum of similar oscillations with periods of $3,4,5$, etc. days, up to chaotic oscillations. Spectral analysis has demonstrated that the harmonic component with a period of two days is most pronounced in the solution of the simplified system corresponding to the period doubling, whereas the amplitudes of the harmonics with periods of $1,0.5,0.25$, etc. days are absent altogether.
Table 1. List of reactions.

\begin{tabular}{|c|c|}
\hline (1) $\mathrm{O}+\mathrm{OH}+\mathrm{M} \rightarrow \mathrm{HO}_{2}+\mathrm{M}$ & (10) $\mathrm{O}+\mathrm{O}_{3} \rightarrow 2 \mathrm{O}_{2}$ \\
\hline (2) $\mathrm{H}+\mathrm{HO}_{2} \rightarrow \mathrm{O}_{2}+\mathrm{H}_{2}$ & (11) $\mathrm{O}+\mathrm{O}+\mathrm{M} \rightarrow \mathrm{O}_{2}+\mathrm{M}$ \\
\hline (3) $\mathrm{OH}+\mathrm{HO}_{2} \rightarrow \mathrm{O}_{2}+\mathrm{H}_{2} \mathrm{O}$ & (12) $\mathrm{O}_{3}+\mathrm{H} \rightarrow \mathrm{O}_{2}+\mathrm{OH}$ \\
\hline (4) $\mathrm{O}+\mathrm{OH} \rightarrow \mathrm{O}_{2}+\mathrm{H}$ & (13) $\mathrm{O}_{3}+\mathrm{OH} \rightarrow \mathrm{O}_{2}+\mathrm{HO}_{2}$ \\
\hline (5) $\mathrm{O}+\mathrm{HO}_{2} \rightarrow \mathrm{O}_{2}+\mathrm{OH}$ & $(14) \mathrm{H}+\mathrm{HO}_{2} \rightarrow 2 \mathrm{OH}$ \\
\hline (6) $\mathrm{O}_{2}+\mathrm{H}+\mathrm{M} \rightarrow \mathrm{HO}_{2}+\mathrm{M}$ & (15) $\mathrm{OH}+\mathrm{OH} \rightarrow \mathrm{O}+\mathrm{H}_{2} \mathrm{O}$ \\
\hline (7) $\mathrm{H}_{2} \mathrm{O}+h v \rightarrow \mathrm{H}+\mathrm{OH}$ & $(16) \mathrm{O}_{3}+h v \rightarrow \mathrm{O}_{2}+\mathrm{O}\left({ }^{1} \mathrm{D}\right)$ \\
\hline (8) $\mathrm{O}_{2}+h v \rightarrow 2 \mathrm{O}$ & (17) $\mathrm{OH}+\mathrm{H}+\mathrm{M} \rightarrow \mathrm{H}_{2} \mathrm{O}+\mathrm{M}$ \\
\hline (9) $\mathrm{O}+\mathrm{O}_{2}+\mathrm{M} \rightarrow \mathrm{O}_{3}+\mathrm{M}$ & (18) $\mathrm{H}+\mathrm{HO}_{2} \rightarrow \mathrm{H}_{2} \mathrm{O}+\mathrm{O}$ \\
\hline
\end{tabular}

$\mathrm{M}$ - a molecule of air.

Additionally, the nonlinearity of this system has a power-law character, which, in principle, is favorable for harmonic expansion. The numerical study of this system with allowance for the diffusion transport reveals the possibility of formation of reaction-diffusion waves which have all the properties of the waves arising in the initial system of equations of the mesospheric photochemical system.

\section{Mesospheric photochemical system and its nonlinear dynamic properties. Methods of model simplification}

The photochemical processes in the mesopause region (80$90 \mathrm{~km}$ ) can be modeled by a mesospheric photochemical system (MPCS) that usually includes 18 most significant reactions with participation of 5 constituents: $\mathrm{O}_{3}, \mathrm{O}, \mathrm{H}, \mathrm{OH}$, and $\mathrm{HO}_{2}$ (see Table 1). The MPCS is subject to external forcing with a period of one day that is associated with the Earth's rotation and has the form of a periodic modulation of photolysis rates of ozone, water vapor, and molecular oxygen.

Temperature, concentrations of air and water vapor molecules, and photolysis rates are basic (control) parameters of the MPCS that have a strong impact on its dynamics. These parameters, in turn, depend on altitude. In the works by Sonnemann et al. (1999) and Sonnemann and Feigin (1999), it was demonstrated that it is sufficient to take this dependence into consideration in the mesopause region only for the air concentration and photolysis rate of molecular oxygen.

The research performed by Fichtelmann and Sonnemann (1992), Sonnemann and Fichtelmann (1997), Feigin et al. (1998), and Konovalov and Feigin (2000) points to a possible nonlinear response of the MPCS to diurnal variations of solar radiation. Analysis within the framework of the zero-dimensional MPCS model revealed a wide spectrum of periodic (subharmonic) and chaotic modes of behavior of minor gas constituents of the mesosphere that are realized depending on the values of control parameters, such as $\mathrm{H}_{2} \mathrm{O}$, 

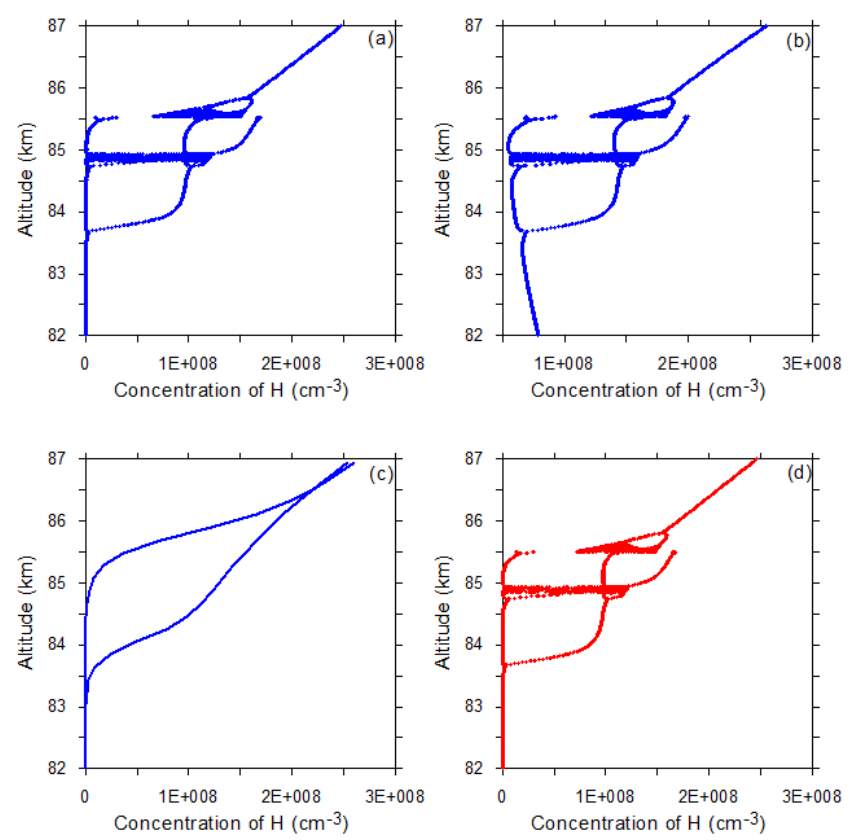

Fig. 1. (a) Bifurcation diagram demonstrating possible modes of MPCS behavior as a function of height; (b) same as in Fig. 1a, but for a different moment of local time; (c) same as in Fig. 1a, but with allowance for vertical eddy diffusion with coefficient $10 \mathrm{~m}^{2} \mathrm{~s}^{-1}$; (d) same as in (a), but without allowance for $\mathrm{H}+\mathrm{HO}_{2} \rightarrow 2 \mathrm{OH}$ and $\mathrm{O}_{3}+\mathrm{OH} \rightarrow \mathrm{O}_{2}+\mathrm{HO}_{2}$ reactions.

temperature and height. Modes of MPCS behavior as a function of height are shown as a bifurcation diagram (BD) in Fig. 1a. Note that time evolution of the MPCS variables is forced; hence, their values depend on local time. One can see in Fig. 1a values of $\mathrm{H}$ concentration acquired at a definite moment of local time corresponding to the end of the night beginning of the day. The sections of the bifurcation diagram, where the dependence of $\mathrm{H}$ concentration on height $(H(z))$ is single-valued, correspond to the oscillations with a period of one day. In the sections where $H(z)$ is a multivalued function, the photochemical oscillations are subharmonic (with periods equal to two, three, four days, etc.) or chaotic. For example, the two-valued function $H(z)$ at heights of about $83.7-84.7 \mathrm{~km}$ corresponds to oscillations with a period of two days. If different moments of local time are chosen (e.g. in the middle of the day), the bifurcation diagram changes quantitatively (Fig. 1b), but qualitatively it fully agrees with the diagram in Fig. 1a.

The nonlinear dynamic mechanism of MPCS response to diurnal variations of solar radiation was described in the work by Konovalov and Feigin (2000). It was shown that this mechanism is closely connected with the system dynamics in the nighttime, when nonlinear relaxation of the great majority of MPCS variables occurs in the absence of a light source. This leads to existence in the phase space of the system of regions with strongly differing image point velocities. It was

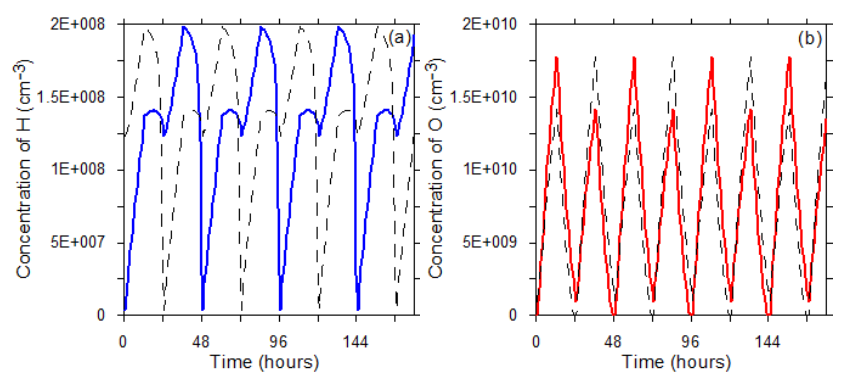

Fig. 2. Oscillations of $\mathrm{H}$ and $\mathrm{O}$ concentrations (a and $\mathbf{b}$, respectively) at a height of $85 \mathrm{~km}$ (solid curves). Dashed curves are for the oscillations of these variables with another value of initial phase.

demonstrated that such a phase space structure at night may result in trajectory scatter.

The analysis of the influence of vertical eddy diffusion on MPCS evolution performed by Sonnemann and Feigin (1999) and Sonnemann et al. (1999) revealed that transport reduces appreciably the spectrum of possible modes of behavior of this system. It was found that for the values of the vertical diffusion coefficient $(5-20) \mathrm{m}^{2} \mathrm{~s}^{-1}$ observed in the mesopause region in summer (e.g. Lubken, 1997), only 2-day MPCS oscillations survive and cover the entire range of heights of the nonlinear response region (see Fig. 1c). It is also clear from this figure that allowance for diffusion leads to pronounced expansion (primarily upwards) of the regions of heights of nontrivial MPCS behavior. We will omit detailed analysis of the causes of this expansion and will only note that that, with the diffusion coupling taken into consideration, the nonlinear dynamic mechanism described by Konovalov and Feigin (2000) is triggered at higher altitudes.

The variation of the other control parameters $\left(\mathrm{H}_{2} \mathrm{O}\right.$ and temperature) within the range of the values typical of the summer mesopause manifests itself into a slight shift of the height of the region where the 2-day oscillations may occur (Kulikov, 2007). These oscillations are illustrated in Fig. 2 for an example of time evolution of $\mathrm{H}$ and $\mathrm{O}$ concentration at a height of $85 \mathrm{~km}$.

The chemical mechanism of the origin of nonlinear behavior of MPCS is conditioned by the well-known catalytic cycle

$$
\begin{aligned}
& \mathrm{HO}_{2}+\mathrm{O} \rightarrow \mathrm{OH}+\mathrm{O}_{2} \\
& \mathrm{OH}+\mathrm{O} \rightarrow \mathrm{H}+\mathrm{O}_{2} \\
& \mathrm{H}+\mathrm{O}_{2}+\mathrm{M} \rightarrow \mathrm{HO}_{2}+\mathrm{M}
\end{aligned}
$$

$2 \mathrm{O} \rightarrow \mathrm{O}_{2}$

that destroys atomic oxygen. The principal feature of this cycle in the conditions of the upper mesosphere is that its rate does not depend on the concentration of the destroyed substance and is determined only by a catalytic agent (atomic hydrogen concentration). As was demonstrated by Konovalov and Feigin (2000), this feature ensures MPCS instability that 
manifests itself as a sequence of period doubling bifurcations and the transition to chaotic behavior as the control parameters are varied (the variation of height in Fig. 1a).

Note that stoichiometric network analysis of the photochemistry in the mesopause region was carried out in the recent work by Hadac and Schreiber (2011). The analysis showed that the chemical mechanism proposed by Konovalov and Feigin (2000) is stable, and a different catalytic cycle

$$
\begin{aligned}
& \mathrm{H}+\mathrm{HO}_{2} \rightarrow 2 \mathrm{OH} \\
& \mathrm{O}_{3}+\mathrm{OH} \rightarrow \mathrm{O}_{2}+\mathrm{HO}_{2} \\
& \mathrm{O}+\mathrm{OH} \rightarrow \mathrm{O}_{2}+\mathrm{H} \\
& \mathrm{O}+\mathrm{O}_{2}+\mathrm{M} \rightarrow \mathrm{O}_{3}+\mathrm{M}
\end{aligned}
$$

$2 \mathrm{O} \rightarrow \mathrm{O}_{2}$

is responsible for the nontrivial nonlinear dynamic properties of MPCS. However, it can be readily demonstrated (for example, by calculating the complete MPCS model) that with formal exclusion from the model of the $\mathrm{H}+\mathrm{HO}_{2} \rightarrow 2 \mathrm{OH}$ or $\mathrm{O}_{3}+\mathrm{OH} \rightarrow \mathrm{O}_{2}+\mathrm{HO}_{2}$ reaction, or exclusion of both these reactions (see Fig. 1d), all the qualitative features of the system are retained and characteristic magnitudes of its variables remain almost unchanged, whereas elimination of any reaction from the Konovalov and Feigin (2000) mechanism completely destroys the MPCS nonlinear response to diurnal variations of light. The point is that, at the heights of the mesopause region, the characteristic rates of the first two reactions of the mechanism proposed by Hadac and Schreiber (2011) are at least an order of magnitude less than the rates of the $\mathrm{HO}_{2}+\mathrm{O} \rightarrow \mathrm{OH}+\mathrm{O}_{2}$ and $\mathrm{O}+\mathrm{OH} \rightarrow \mathrm{O}_{2}+$ $\mathrm{H}$ reactions. Therefore, the $\mathrm{H}+\mathrm{HO}_{2} \rightarrow 2 \mathrm{OH}$ and $\mathrm{O}_{3}+\mathrm{OH} \rightarrow$ $\mathrm{O}_{2}+\mathrm{HO}_{2}$ reactions are secondary for the mesospheric photochemistry and do not affect its nonlinear dynamic properties.

It is worthy of notice that 2-day oscillations are forced and, depending on initial conditions, there may exist two solutions differing by initial phases only (Kulikov and Feigin, 2005). This taken into account, the initial phase of the 2-day oscillations may be represented as a sum of three quantities:

$\varphi_{p h}=\varphi_{0} / 2+\hat{\varphi}+\alpha$.

Here, $\alpha$ is the latitude-dependent constant, $\varphi_{0}$ is the phase of external, one day periodic forcing, and $\hat{\varphi}$ may take on one of the two values, $\pi$ or $2 \pi$, depending on initial conditions. Figure 2 shows two possible MPCS solutions corresponding to these phase values. It is apparent that this feature can manifest itself in the dependence of the characteristics of the 2-day oscillations on the horizontal coordinates. The phase distribution of the 2-day oscillations may be arbitrary, and distribution of the concentrations of minor gas constituents may be significantly inhomogeneous. In particular, appreciable gradients in the concentrations of minor gas constituents of the MPCS arise in the neighborhood of abrupt jumps in the phase of the 2-day oscillations. Note that in the zonal direction (around the latitudinal circle), there exists natural inhomogeneity in the phase distribution of diurnal variations of solar radiation that is caused by the dependence of the sunrise and sunset moments on the zonal coordinate $x$ :

$\varphi_{0}=2 \pi x / L$,

where $L$ is the length of the corresponding latitudinal circle. With Eq. (2) taken into account, Equaton (1) for the phase of the 2-day oscillations takes on the form

$\varphi_{p h}(x)=\pi x / L+\hat{\varphi}(x)$.

It follows from that, in particular, that even in the case of the homogeneous zonal distribution of the initial phases of subharmonic oscillations $(\hat{\varphi}(x) \equiv$ const), at least one phase jump exists in the zonal circle. The presence of sharp gradients in the horizontal distribution of concentrations demands allowance for the horizontal eddy diffusion that "smooths" the phase and concentration gradients. The resulting scale of horizontal inhomogeneity of the oscillatory phase and concentration of minor gas constituents is evidently determined by the balance between the photochemical and diffusion processes. The numerical simulation performed by Kulikov and Feigin $(2004,2005)$ taking into consideration the horizontal diffusion revealed that the diffusion transport gives rise to reaction-diffusion waves in the form of propagating fronts and pulses. These waves are phase waves and manifest themselves in a uniform, constant-rate displacement of sharp jumps of the initial phase along the latitudinal circle. The waves are caused by the presence of the zonal inhomogeneity of the phase of the diurnal light variations: $\varphi_{0}=2 \pi x / L$. In the case of uniform spatial distribution of the phase of diurnal light variations, sharp changes in the initial phase turn out to be stationary. It is shown that the propagation velocity of such waves is directly proportional to the horizontal diffusion coefficient and the gradient of the spatial nonuniformity of the phase of diurnal variations of light along the latitudinal circle, i.e. $L^{-1}$.

Feigin and Konovalov (1996) and Feigin et al. (1998) showed that basic dynamic models (BDMs) are a good tool for studying the evolution of atmospheric photochemical systems. Within the considered region of the parameter values, BDMs retain basic qualitative and quantitative properties of a "complete" system and include a minimum possible number of dynamic variables described by differential equations. The key idea of constructing such models is to divide variables of the system (concentrations of chemical constituents) into two groups according to the relationship between the characteristic time of their evolution, " $\tau$ ", and the time scale of the studied phenomenon, $\tau_{0}$. Variables of the first group are "fast", i.e. $\tau \ll \tau_{0}$. Taking into consideration the dissipativity of atmospheric photochemical systems, they are supposed to be in the state of instantaneous stable equilibrium. In other words, it is supposed that the relation

$\frac{d}{d t} n \ll I, S$ 
is fulfilled in the chemical balance equation of such a fast component. Here, $I$ and $S$ are the total photochemical source and sink, respectively. Thus, "instantaneously equilibrium" values of fast variables are determined from a system of algebraic equations obtained by zeroing the time derivatives in the corresponding equations of chemical kinetics of the initial (complete) system. These values are generally functions of parameters and of the slow (dynamic) variables referred to by the second group whose evolution with characteristic time $\tau \approx \tau_{0}$ is described by differential equations.

A zero-dimensional basic dynamic model of MPCS was constructed by Feigin et al. (1998). In this model, O and $\mathrm{H}$ concentrations are dynamic variables (with characteristic times equal to $5 \times 10^{4}-10^{5} \mathrm{~s}$ ) found from a system of two first-order differential equations:

$$
\begin{aligned}
& \frac{d x_{1}}{d t}=-\alpha x_{2}-(1-s(t)) \mu x_{1}+\delta s(t) \\
& \frac{d x_{2}}{d t}=-\beta \frac{x_{2}^{2}}{x_{1}^{2}}-\sigma \frac{x_{2}^{2}}{x_{1}}+\gamma s(t),
\end{aligned}
$$

where

$s(t)=\left\{\begin{array}{ll}1, & t \in[\tau n ; \tau n+\tau / 2] \\ 0, & t \in[\tau n+\tau / 2 ; \tau(n+1)]\end{array} \quad n=1,2, \ldots\right.$,

$\alpha=2 R_{6} \cdot \mathrm{O}_{2} \cdot \mathrm{M}, \quad \mu=R_{9} \cdot \mathrm{O}_{2} \cdot \mathrm{M}, \quad \delta=2 R_{8} \cdot \mathrm{O}_{2}$,

$\beta=2 \frac{R_{3} R_{6}^{2}}{R_{5} R_{4}} \cdot \mathrm{O}_{2}^{2} \cdot \mathrm{M}^{2}, \quad \sigma=2 \frac{R_{6}\left(R_{2}+R_{18}\right)}{R_{5}} \cdot \mathrm{O}_{2} \cdot \mathrm{M}$,

$\gamma=2 R_{7} \cdot r \cdot \mathrm{M}$,

where $r$ is water vapor concentration (ppmv), $x_{1}$ and $x_{2}$ are $\mathrm{O}$ and $\mathrm{H}$ concentrations, respectively, and $R_{1-18}$ are the constants of the reactions listed in Table 1. Following the works by Sonnemann et al. (1999) and Sonnemann and Feigin (1999), we will further take into consideration the following dependences of parameters on height:

$\mathrm{O}_{2}, \mathrm{M} \sim e^{-z / H_{a}}, \quad R_{8} \sim e^{-z /\left(2 H_{a}\right)}, H_{a}=6 \mathrm{~km}$.

The concentrations of the remaining three constituents $\left(\mathrm{O}_{3}, \mathrm{OH}\right.$, and $\left.\mathrm{HO}_{2}\right)$ are "fast" variables that are found from the system of algebraic equations

$\mathrm{O}_{3}=\left\{\begin{array}{l}\frac{R_{9} x_{1}}{R_{16}} \cdot \mathrm{O}_{2} \cdot \mathrm{M}-\text { day } \\ \text { const }- \text { night }\end{array}, \mathrm{OH}=\frac{R_{6} x_{2}}{R_{4} x_{1}} \cdot \mathrm{O}_{2} \cdot \mathrm{M}, \mathrm{HO}_{2}=\frac{R_{6} x_{2}}{R_{5} x_{1}} \cdot \mathrm{O}_{2} \cdot \mathrm{M}\right.$.

The modes of the BDM behavior are shown in Fig. 3a.

Note that despite the minimum ${ }^{1}$ possible number of differential equations, System (4) is still rather complicated. Therefore, it is almost impossible to use its initial form for analytical study of the mechanism of generation of two-day oscillations. First, the external periodic forcing is strong, so the MPCS variables can change by several orders of magnitude during one day. In other words, the time evolution of these variables is modulated significantly by a one-day period (see Fig. 2). Second, this forcing is significantly nonharmonic (meandering), which results in appearance in the

\footnotetext{
1 needed for formation of the nontrivial behavior illustrated in
} Fig. 3.
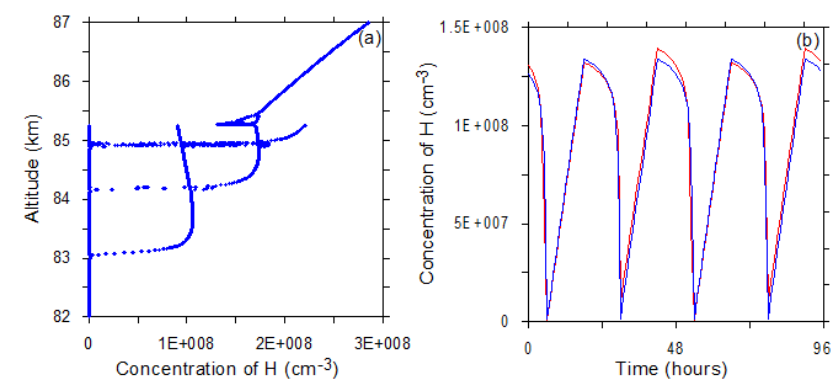

Fig. 3. (a) Bifurcation diagram demonstrating modes of behavior of the initial system of equations for MPCS BDM as a function of height; (b) oscillations of $\mathrm{H}$ concentration at $83 \mathrm{~km}$ (blue curve) and $83.06 \mathrm{~km}$ (red curve).

oscillation spectrum of the MPCS variables of harmonics with periods of $1 / 2,1 / 4$, etc. days, whose amplitudes decrease relatively slowly depending on their number. Third, the fractional power nonlinearity in the second equation of System (4) makes it virtually impossible to perform harmonic expansion, which would allow one to obtain equations for the component with a period of two days. Therefore, we used the methods and approaches widely employed for solution of such problems in nonlinear laboratory chemistry (Field and Burger, 1985). To begin with, we analyzed individual terms of the equations of System (4) so as to single out the most important terms that determine the nonlinear response to the external periodic forcing. The remaining, less significant terms were omitted. Apparently, this simplification resulted in both the quantitative and qualitative changes - specifically, the boundaries of the nonlinear response region, bifurcation types, and their order changed. However, the truncated system still demonstrated the oscillations with a two-day period, which is important for further analysis. At the second stage, we expanded the obtained system of equations near the period doubling threshold. It can be seen from Fig. 3a that the excitation of the two-day regime is supercritical, i.e. near the $83-\mathrm{km}$ height, and the solution differs little from the 1-day oscillations (see Fig. 3b). This means that near this threshold the solution can be represented as a sum of the main solution with a period of one day and a small addition corresponding to the new period. The equations for this addition can be found by linearization of the simplified system of equations.

\section{Development of the applicable model for analytical analysis. Linearization near the period doubling threshold}

\subsection{Simplification of the model}

At the first stage of simplification of System (4), the function $s(t)$ was replaced by a sum of two harmonics that correspond to the zeroth and main frequency of the external forcing, so 

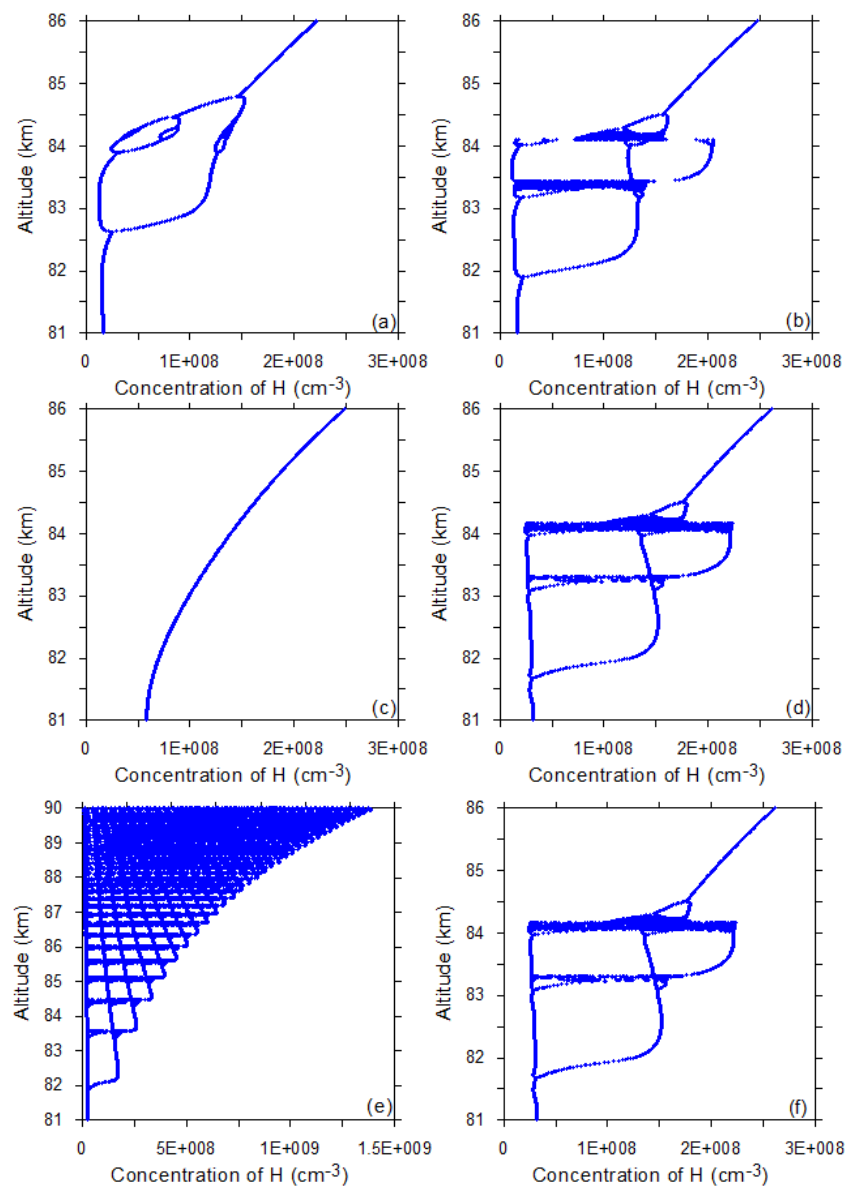

Fig. 4. (a) Bifurcation diagram demonstrating modes of behavior of System (6) as a function of height; (b) as in (a), but for System (7); (c) as in (a), but for System (7) without allowance for the external periodic forcing in the first equation of the system; (d) as in (a), but for System (7) without allowance for the external periodic forcing in the second equation of the System; (e) as in (a), but for System (8); (f) as in (a), but for System (9).

as to decrease the amplitudes of harmonics of the external periodic forcing (with periods of $1 / 2,1 / 4$, etc. days) in the spectrum of oscillations of the MPCS variables:

$$
\begin{aligned}
& \frac{d x_{1}}{d t}=-\alpha x_{2}-\frac{1}{2}\left(1-\sin \frac{2 \pi t}{T}\right) \mu x_{1}+\frac{\delta}{2}+\frac{\delta}{2} \sin \frac{2 \pi t}{T} \\
& \frac{d x_{2}}{d t}=-\beta \frac{x_{2}^{2}}{x_{1}^{2}}-\sigma \frac{x_{2}^{2}}{x_{1}}+\frac{\gamma}{2}+\frac{\gamma}{2} \sin \frac{2 \pi t}{T}
\end{aligned}
$$

The bifurcation diagram for this system is shown in Fig. 4a. As expected, with such a substitution the main property of the initial system (nonlinear response) is retained, despite the significant quantitative and qualitative changes compared with the BD shown in Fig. 3. Note, first, that the constant terms in the right-hand sides of Eq. (6) ensure nonnegativity of the variables, so it is meaningless to omit them. Second, the term $-\alpha x_{2}$ in the first equation is of key importance, as it ensures feedback with the variable $x_{2}$ without which the nonlinear response is impossible at any parameter values of System (6). The important fact is that this term implies the presence of the catalytic cycle of atomic oxygen destruction in the photochemical system under consideration. The destruction rate is determined by the concentration of atomic hydrogen, rather than by the concentration of the destroyed substance. As was shown by Konovalov and Feigin (2000), this cycle ensures the chemical mechanism of formation of the nonlinear response.

At the second stage, we omitted the term responsible for the linear periodic dissipation of the variable $x_{1}$ :

$$
\begin{aligned}
& \frac{d x_{1}}{d t}=-\alpha x_{2}+\frac{\delta}{2}+\frac{\delta}{2} \sin \frac{2 \pi t}{T} \\
& \frac{d x_{2}}{d t}=-\beta \frac{x_{2}^{2}}{x_{1}^{2}}-\sigma \frac{x_{2}^{2}}{x_{1}}+\frac{\gamma}{2}+\frac{\gamma}{2} \sin \frac{2 \pi t}{T}
\end{aligned}
$$

The BD for this system is shown in Fig. 4b. Note that the behavior of System (7) correlates with the initial system (in terms of their spectrum and order) much better than in the previous case.

At the third stage, we analyzed whether it is necessary to take into account external periodic forcing simultaneously for both variables of System (7). It was found that, if the term responsible for the forcing is omitted in the first equation, the nonlinear response disappears (see Fig. 4c), whereas its presence in the equation for the term $x_{2}$ is less significant (see Fig. 4d) and may be neglected.

Finally, we studied the importance of both types of nonlinearity and considered two systems of equations, respectively:

$$
\begin{aligned}
& \frac{d x_{1}}{d t}=-\alpha x_{2}+\frac{\delta}{2}+\frac{\delta}{2} \sin \frac{2 \pi t}{T} \\
& \frac{d x_{2}}{d t}=-\beta \frac{x_{2}^{2}}{x_{1}^{2}}+\frac{\gamma}{2}
\end{aligned}
$$

and,

$\frac{d x_{1}}{d t}=-\alpha x_{2}+\frac{\delta}{2}+\frac{\delta}{2} \sin \frac{2 \pi t}{T}$

$\frac{d x_{2}}{d t}=-\sigma \frac{x_{2}^{2}}{x_{1}}+\frac{\gamma}{2}$.

It was found that both nonlinearities lead to nonlinear response (see Fig. 4e and f), but the behavior of System (9) correlates with the initial System (4) much better in terms of their spectrum and order. Therefore, it was chosen for the further analysis. In the case of System (8), the nonlinear response proved to be height-unbounded from above, which allows (see Fig. 4e) generation of multiperiodic oscillations with a period of tens of days and more. Specifically, at a height of $90 \mathrm{~km}$, this type of nonlinearity leads to oscillations with a period of 39 days, which is not observed in the initial system.

Let us pass over to the dimensionless variables of System (9). We use the following substitution:

$x=\frac{2 \alpha}{\delta} x_{1}, \quad y=\frac{2 \gamma \alpha^{2}}{\sigma \delta^{2}} x_{2} ; \quad \tau=\frac{t}{T}$, 


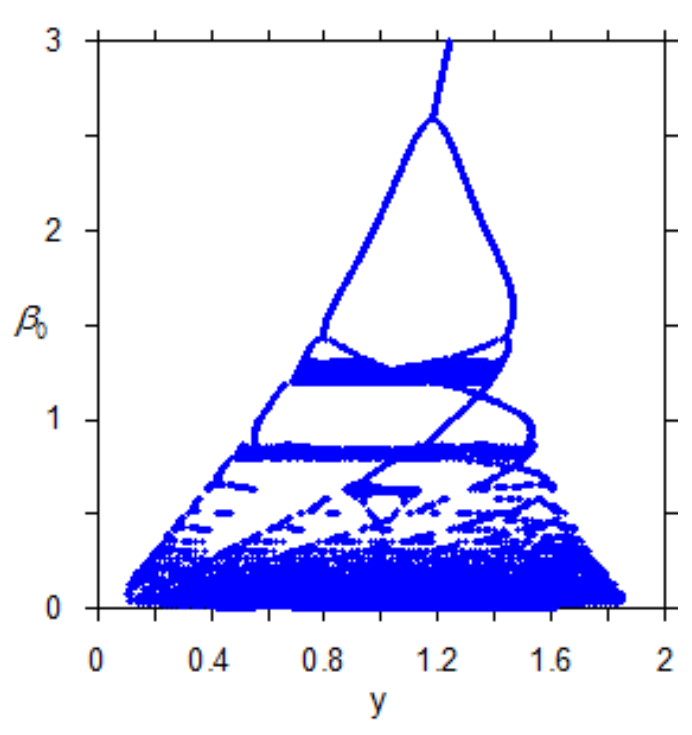

Fig. 5. Bifurcation diagram demonstrating modes of behavior of the system of Eq. (10).

thus appreciably reducing the number of parameters:

$$
\begin{aligned}
& \frac{d x}{d \tau}=\alpha_{0}(1+\sin (2 \pi \tau)-y) \\
& \frac{d y}{d \tau}=\beta_{0}\left(1-\frac{y^{2}}{x}\right)
\end{aligned}
$$

where $\alpha_{0}=\frac{\gamma \alpha^{2}}{\sigma \delta} T, \beta_{0}=\frac{\gamma \alpha T}{\delta}$. In what follows, for the sake of certainty and simplicity, we will regard these parameters to be independent and use, e.g. $\beta_{0}$ as the control parameter. Figure 5 shows an example of the bifurcation diagram of System (10) at $\alpha_{0}=10$. One can see that at this value of $\alpha_{0}$, the generation of oscillations with a doubled period starts near $\beta_{\text {bif }} \approx 2.6$.

\subsection{Linearization near the period doubling threshold}

We linearize System (10) near the bifurcation value of the parameter $\beta_{\text {bif }} \approx 2.6$, making use of the fact that the new solution with the doubled period actually differs little from the solution with a period of 1 . To do so, we represent the solution in the form $x=x_{1}+x_{2}, y=y_{1}+y_{2},\left|x_{2}\right|,\left|y_{2}\right| \ll$ $\left|x_{1}\right|,\left|y_{1}\right|$, and in what follows we will regard $\left(x_{1}, y_{1}\right)$ to be a solution of the system before the bifurcation $\left(\beta_{0}>\beta_{\text {bif }}\right)$ and periodic with a period of external forcing. Let us expand System (10) in a series to the first order in small parameters $x_{2} / x_{1}, y_{2} / y_{1}$ :

$$
\begin{aligned}
& \frac{d x_{1}}{d \tau}+\frac{d x_{2}}{d \tau}=\alpha_{0}\left(1+\sin (2 \pi \tau)-y_{1}-y_{2}\right) \\
& \frac{d y_{1}}{d \tau}+\frac{d y_{2}}{d \tau}=\beta_{0}\left(1-\frac{y_{1}^{2}}{x_{1}}+\frac{y_{1}^{2}}{x_{1}^{2}} x_{2}-2 \frac{y_{1}}{x_{1}} y_{2}\right) .
\end{aligned}
$$

By excluding the higher terms responsible for the generation of $\left(x_{1}, y_{1}\right)$, we obtain a linear system of equations for

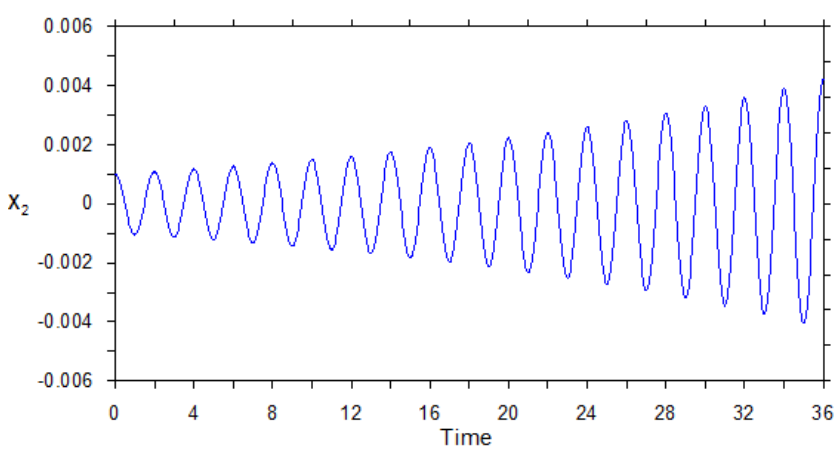

Fig. 6. Time evolution of the solution of Eq. (12) obtained for parameter $\beta_{0}$ slightly less than the threshold value.

$\left(x_{2}, y_{2}\right)$

$\frac{d x_{2}}{d \tau}=-\alpha_{0} y_{2}$

$\frac{d y_{2}}{d \tau}=\beta_{0}\left(\frac{y_{1}^{2}}{x_{1}^{2}} x_{2}-2 \frac{y_{1}}{x_{1}} y_{2}\right)$.

Here, $\frac{y_{1}^{2}}{x_{1}^{2}}$ and $\frac{y_{1}}{x_{1}}$ are periodic functions with a period of external forcing, therefore they may be expanded in the Fourier series:

$$
\begin{aligned}
& \frac{y_{1}^{2}}{x_{1}^{2}}=\left(\frac{y_{1}^{2}}{x_{1}^{2}}\right)_{0}+\left(\frac{y_{1}^{2}}{x_{1}^{2}}\right)_{1} \sin (2 \pi \tau+\varphi)+\sum \ldots \\
& \frac{y_{1}}{x_{1}}=\left(\frac{y_{1}}{x_{1}}\right)_{0}+\left(\frac{y_{1}}{x_{1}}\right)_{1} \sin (2 \pi \tau+\varphi)+\sum \ldots
\end{aligned} .
$$

The above equations contain harmonics of the external periodic forcing under the summation sign. It is evident that within the approximation to which System (11) corresponds, these harmonics with periods of $0.5,0.25,0.125$, etc. days cannot result in the excitation of a solution with a period equal to 2. It means that in the first order of smallness, these harmonics can be omitted, and specific values of the constants $\left(\frac{y_{1}^{2}}{x_{1}^{2}}\right)_{0},\left(\frac{y_{1}^{2}}{x_{1}^{2}}\right)_{1},\left(\frac{y_{1}}{x_{1}}\right)_{0},\left(\frac{y_{1}}{x_{1}}\right)_{1}$ can be determined from the Fourier expansion of the numerical solution of System (10) near the bifurcation threshold $\beta_{\text {bif }} \approx 2.6$. As a result, System (11) is easily rewritten in the form of one equation:

$$
\begin{aligned}
\frac{d^{2} x_{2}}{d \tau^{2}} & +2 \beta_{0}\left(\left(\frac{y_{1}}{x_{1}}\right)_{0}+\left(\frac{y_{1}}{x_{1}}\right)_{1} \sin (2 \pi \tau+\varphi)\right) \frac{d x_{2}}{d \tau} \\
& +\alpha_{0} \beta_{0}\left(\left(\frac{y_{1}^{2}}{x_{1}^{2}}\right)_{0}+\left(\frac{y_{1}^{2}}{x_{1}^{2}}\right)_{1} \sin (2 \pi \tau+\varphi)\right) x_{2}=0 .
\end{aligned}
$$

This is a linear oscillator equation with parametric action and periodic dissipation. Figure 6 shows the time evolution of the solution of this equation, which was obtained for $\beta_{0}=$ 2.55. One can see that this solution represents the needed oscillations with a period equal to 2 , whose amplitude grows exponentially with time. The performed numerical analysis shows, first, that if the values of the constants in Eq. (12) 
are determined for $\beta_{0}>\beta_{\text {bif }}$, the oscillations with a period equal to 2 are not excited. Second, the obtained equation can be made much simpler, as neither the constant portion of the frequency of our linear oscillator nor the periodic dissipation lead to excitation of the solution that we seek and, hence, can be omitted. The obtained equation

$$
\frac{d^{2} x_{2}}{d \tau^{2}}+\lambda \sin (\omega \tau+\varphi) x_{2}=0, \quad \lambda=\alpha_{0} \beta_{0}\left(\frac{y_{1}^{2}}{x_{1}^{2}}\right)_{1}
$$

is a particular case of the Mathieu equation. To the first approximation (neglecting the harmonics $\frac{3 \omega}{2}, \frac{5 \omega}{2}$, etc.), the solution can be written in the analytical form:

$$
\begin{aligned}
x_{2} & =A \exp \left(i \frac{\omega}{2} \tau+\tau \sqrt{\frac{\lambda}{2}-\frac{\omega^{2}}{4}}+i \frac{\varphi}{2}\right) \\
& +B \exp \left(i \frac{\omega}{2} \tau-\tau \sqrt{\frac{\lambda}{2}-\frac{\omega^{2}}{4}}+i \frac{\varphi}{2}\right)+k . c .
\end{aligned}
$$

One can make two conclusions from this expression. First, the increment of oscillations at half frequency arises when $\lambda>\omega^{2} / 2$. Second, since the phase $\varphi$ of the photochemical oscillations with the frequency of the external forcing is related linearly by the intrinsic phase $\varphi_{0}$ of this forcing ( $\varphi=\varphi_{0}+$ const), the phase of oscillations with a doubled period is $\varphi_{2}=\varphi_{0} / 2+$ const. Note, first, that in the initial system of Eq. (4), this relationship between the phases of the two-day oscillations and diurnal variations of solar radiation is ambiguous, as was found numerically by Kulikov and Feigin $(2004,2005)$. Second, it follows from this phase relationship that, taking into consideration the invariance of $\varphi_{0} \rightarrow \varphi_{0}+2 \pi$, the phase of the excited subharmonic oscillations can take on two values differing by $\pi$, depending on the initial conditions.

\section{A system of two differential equations with power-law nonlinearity and reaction-diffusion waves}

\subsection{A system of equations with power-law nonlinearity}

It was shown in the previous section that the period doubling is described by a particular case of the Mathieu equation, where unlimited instability of the first subharmonic of this forcing arises at a sufficiently high amplitude of external forcing $\left(\lambda>\omega^{2} / 2\right)$. However, in the initial model, the amplitude of the two-day oscillations is limited. Therefore, for further analysis we found a simple mechanism that would stabilize the exponential growth of the Mathieu equation. For this, System (10) was expanded in series up to higher orders of smallness, and the best-fit (in terms of limitation of instability) nonlinearity in the form $-x^{2} y$ was found enumeratively. Thus, we obtained a system of two differential equations with power-law nonlinearity required for analytical study of the

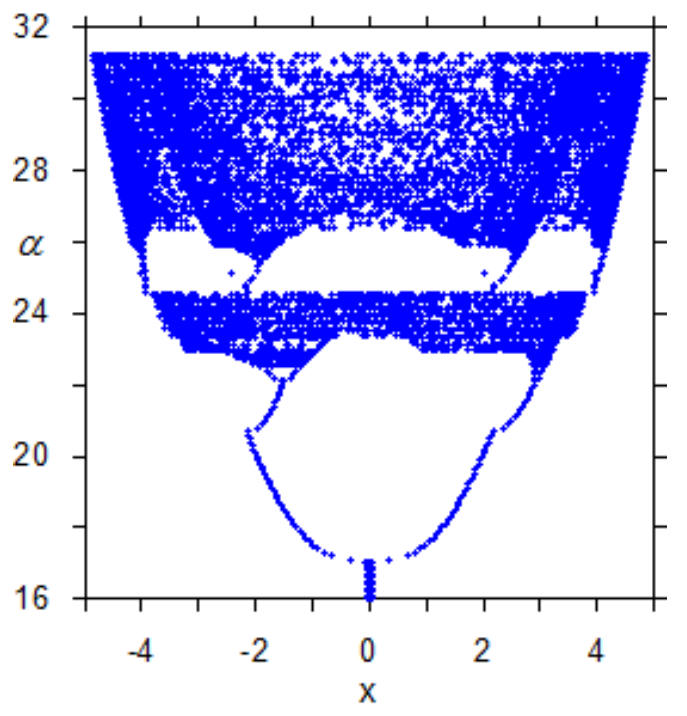

Fig. 7. Bifurcation diagram demonstrating modes of behavior of the system of Eq. (15) for $\omega=2 \pi$.

effect of formation of reaction-diffusion waves in the mesospheric photochemical system:

$\frac{d x}{d t}=-y$
$\frac{d y}{d t}=\alpha \sin (\omega t+\varphi) x-x^{2} y$.

Figure 7 shows the BD of this system as a function of the parameter $\alpha$, demonstrating the possibility of a wide spectrum of non-trivial subharmonic and chaotic behavior. One can see that the nonlinear response arises in this system at $\alpha>17$, which is a little lower than the period doubling threshold $\alpha_{\text {bif }} \approx 19.74$ obtained from the analytical formula of Eq. (14). Such a small difference is due to the neglect of the weak influence of harmonics with semi-integral periods $\frac{3 \omega}{2}, \frac{5 \omega}{2}$, etc. in Eq. (14). The time evolution of the variables in System (15) and the respective time spectra corresponding to the oscillations with a period equal to 2 are presented in Fig. 8. One can see, first, that the oscillations with half-frequency $\left(\frac{\omega}{2}\right)$ have the highest amplitude, whereas the amplitudes of the other harmonics rapidly decrease as their number increases. Therefore, one can neglect their influence in the first order of smallness. Second, another favorable circumstance is absence of the frequency of external periodic forcing $(\omega)$ and its harmonics $(2 \omega, 3 \omega, 4 \omega \ldots)$ in the presented spectra.

\subsection{Reaction-diffusion waves}

For verification of the possibility of simulating the reactiondiffusion waves of the mesospheric photochemistry using System (15), we considered a continuous chain of such nonlinear oscillators with diffusion coupling and with linear spatial inhomogeneity of the phase of external periodic forcing: 

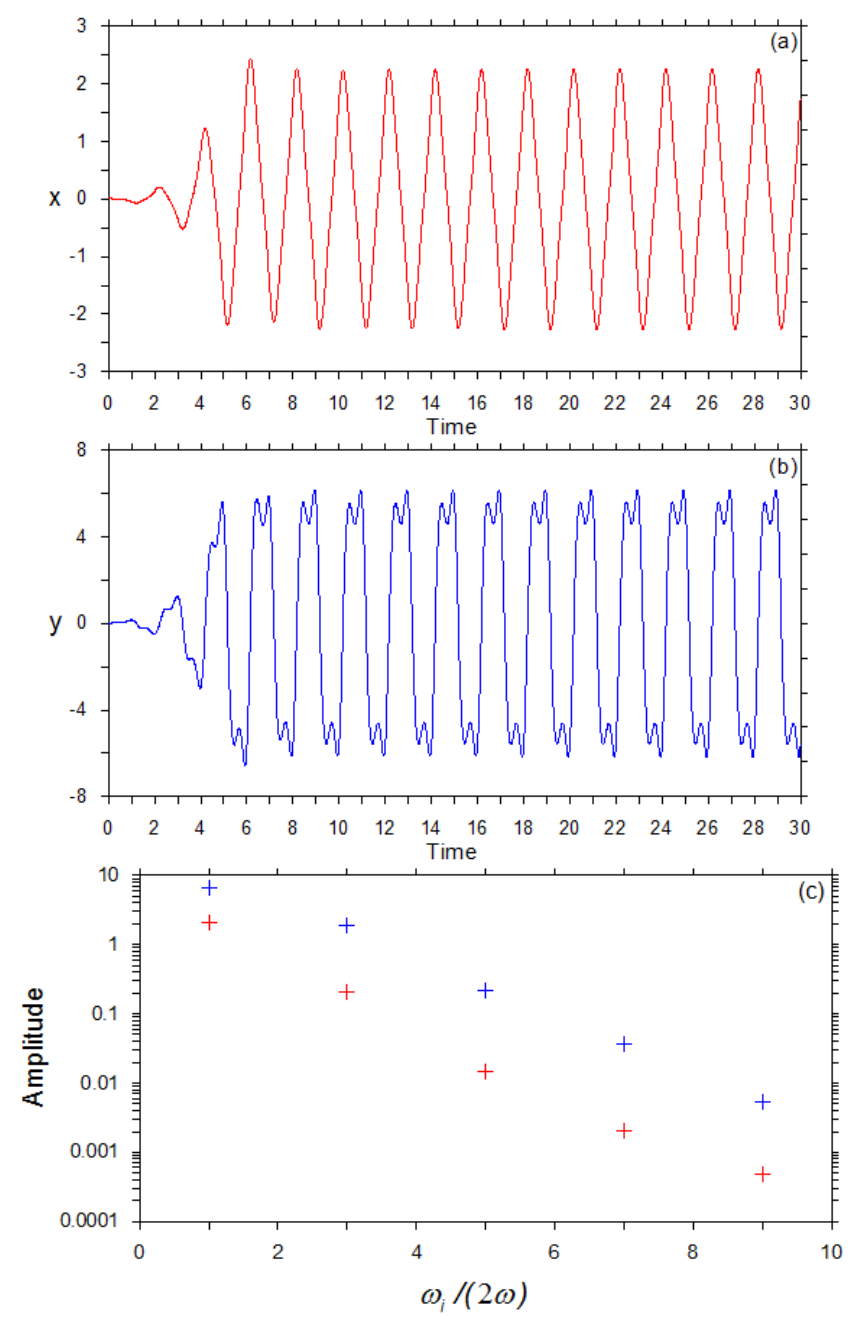

Fig. 8. (a-b) Time evolution of the variables in System (15) for $\alpha=20$; (c) spectra of harmonic oscillations of the variables.

$$
\begin{aligned}
& \frac{\partial x}{\partial t}=-y+D \frac{\partial^{2} x}{\partial l^{2}} \\
& \frac{\partial y}{\partial t}=\alpha \sin \left(\omega t+\frac{2 \pi}{L} l\right) x-x^{2} y+D \frac{\partial^{2} y}{\partial l^{2}} .
\end{aligned}
$$

Here, $D$ is the diffusion coefficient and $L$ is the length of the oscillator chain, i.e. the length of the corresponding "circle" formed by the spaced apart oscillators. It follows from the conclusions of Sect. 3 that, even in the case of uniform spatial distribution $\left(\varphi_{0}(x) \equiv\right.$ const) of the initial phases of subharmonic oscillations with a period equal to 2 , there exists at least one sharp phase change. We performed numerical simulation of System (16) with periodic boundary conditions and found the reaction-diffusion waves (see Fig. 9a) possessing all the properties typical of the waves in the initial system of Eq. (4). First, these waves have a strictly defined direction of propagation. Second, the transition of these waves through a fixed point in space (i.e. through a specific oscillator) leads to a change in the phase of local oscillations by
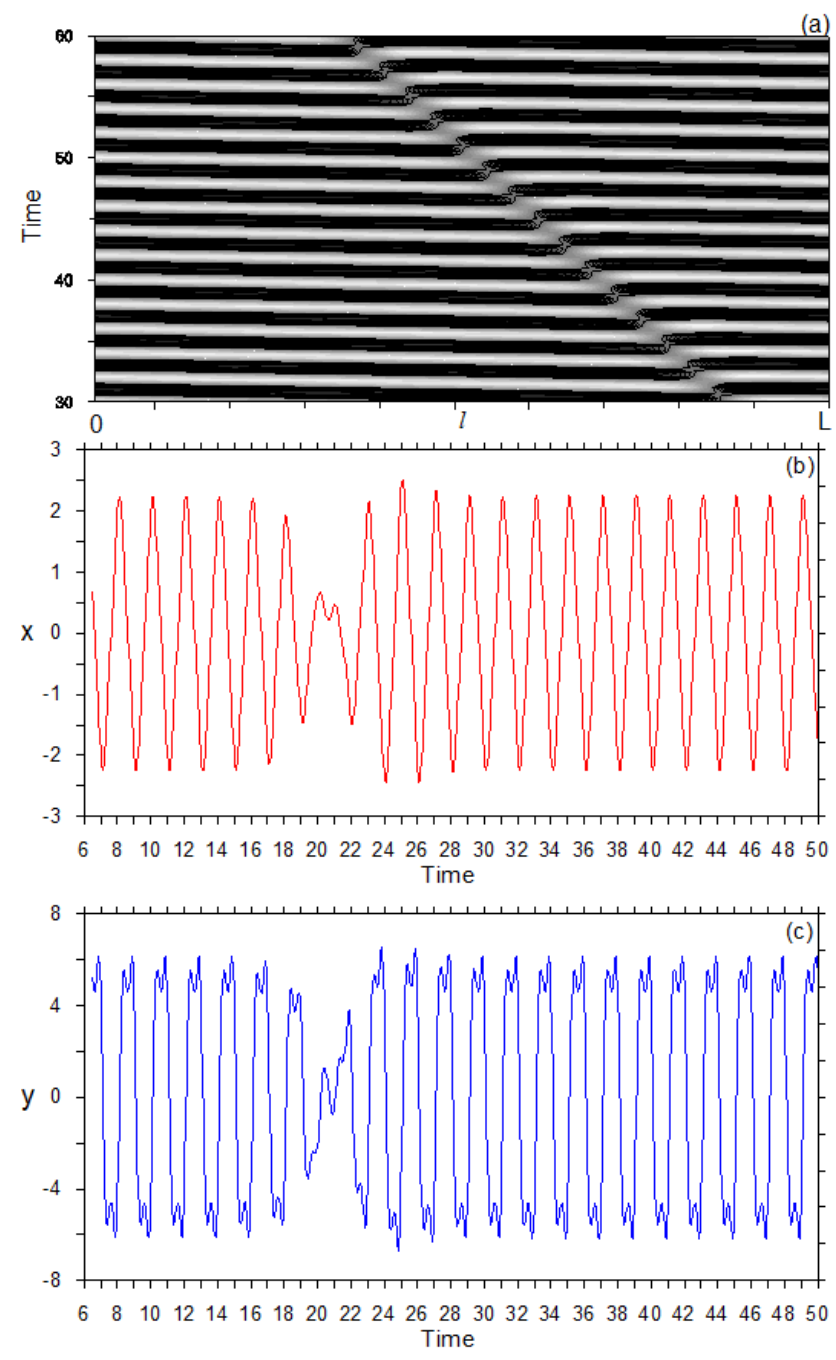

Fig. 9. (a) Spatio-temporal evolution of the solution of system (16) allowing for diffusion. The front of the phase jump propagates along the zonal circle with constant velocity. Dark and light regions correspond to the solution with phases differing by $\pi$; (b-c) local evolution of the variables in system (16) under the conditions of phase front propagation.

$\pi$ (see Fig. 9b-c). Third, when the external forcing has uniform phase distribution, the phase changes are stationary, i.e. no waves are formed. Fourth, the wave propagation velocity is directly proportional to $D$ and inversely proportional to $L$ to a good accuracy.

\section{Discussion and conclusion}

Thus, in this work we have studied analytically the mechanism of generation of two-day photochemical oscillations in the mesopause region. To do so, we simplified the initial System of Eq. (4) and reduced it to system (9) that reproduces sufficiently well the principal qualitative features of 
the initial model. The linearization near the period doubling threshold demonstrated that the mechanism of generation is described by the particular case of the Mathieu equation in which, at high enough amplitude of external forcing, the first subharmonic of the forcing becomes unstable.

Note, first, that the obtained system of Eq. (9) takes into consideration only 7 photochemical reactions:

$$
\begin{aligned}
& \mathrm{H}+\mathrm{HO}_{2} \rightarrow \mathrm{O}_{2}+\mathrm{H}_{2} \\
& \mathrm{O}+\mathrm{OH} \rightarrow \mathrm{O}_{2}+\mathrm{H} \\
& \mathrm{O}+\mathrm{HO}_{2} \rightarrow \mathrm{O}_{2}+\mathrm{OH} \\
& \mathrm{O}_{2}+\mathrm{H}+\mathrm{M} \rightarrow \mathrm{HO}_{2}+\mathrm{M} \\
& \mathrm{H}_{2} \mathrm{O}+h v \rightarrow \mathrm{H}+\mathrm{OH} \\
& \mathrm{O}_{2}+h v \rightarrow 2 \mathrm{O} \\
& \mathrm{H}+\mathrm{HO}_{2} \rightarrow \mathrm{H}_{2} \mathrm{O}+\mathrm{O}
\end{aligned}
$$

Second, for nontrivial nonlinear dynamic properties of System (9) to arise, harmonic action should be taken into account only for the $\mathrm{O}_{2}+h v \rightarrow 2 \mathrm{O}$ reaction. We carried out a special numerical-analytical analysis of System (7) aimed at understanding why allowance for periodic action in the equation for variable $x_{2}$ only leads to disappearance of nonlinear response. We considered the following system of equations:

$$
\begin{aligned}
& \frac{d x_{1}}{d t}=-\alpha x_{2}+\frac{\delta}{2} \\
& \frac{d x_{2}}{d t}=-\beta \frac{x_{2}^{2}}{x_{1}^{2}}-\sigma \frac{x_{2}^{2}}{x_{1}}+\frac{\gamma}{2}+\frac{\gamma}{2} \sin \frac{2 \pi t}{T} .
\end{aligned}
$$

System (17) was expanded like in Sect. 3 and the Mathieu equation for a two-day harmonic was obtained. The numerical analysis revealed that the solution of this equation is stable and has no oscillations with a period of 2 days throughout the simulated range of heights. In other words, allowance for periodic action only in the equation for $x_{2}$ does not lead to appearance of an increment for this harmonic.

Note that the studied mechanism is also responsible for all further period doubling bifurcations and subsequent supercritical excitation of subharmonic oscillations with periods of 4, 8, 16, etc. days. Indeed,

1. if we consider System (10) near the excitation threshold of the oscillations with, e.g. a period of 4 days (see Fig. 5),

2. if the solution is represented in the form $x=x_{1}+x_{2}+$ $x_{4}, y=y_{1}+y_{2}+y_{4},\left|x_{4}\right|,\left|y_{4}\right|<<\left|x_{1}+x_{2}\right|,\left|y_{1}+y_{2}\right|$, where $\left(\mathrm{x}_{1}+\mathrm{x}_{2}, \mathrm{y}_{1}+\mathrm{y}_{2}\right)$ is the solution of the system before the bifurcation and includes the harmonics with frequencies $\omega^{2}$ and $\frac{\omega}{2}$, respectively,

3. if all the required small parameter and Fourier series expansions are performed in accordance with the procedures described in Sect. 3, and

\footnotetext{
2 frequency of the external periodic forcing.
}

4. if we single out the terms in resonance with the frequency we are interested in,

then we will obtain an equation describing the excitation of solutions with frequency $\omega / 4$ :

$\frac{d^{2} x_{4}}{d \tau^{2}}+\lambda \sin \left(\frac{\omega}{2} \tau+\frac{\varphi_{0}}{2}+\right.$ const $) x_{4}=0$,

where $\varphi_{0}$ is the phase of external periodic forcing. Note that from the formal viewpoint, natural oscillations with frequency $\omega / 2$ act as the "external" forcing required to excite these oscillations, rather than as the initial forcing with frequency $\omega$. For excitation of the oscillations with frequency $\omega / 8$, we obtain the equation $\frac{d^{2} x_{8}}{d \tau^{2}}+\lambda \sin \left(\frac{\omega}{4} \tau+\frac{\varphi_{0}}{4}+\right.$ const $) x_{8}=0$, etc. Here, the following regularity is easily observed. The phase of subharmonic oscillations with a period of $N$ days is represented as a sum of three values, $\varphi_{0} / N+\varphi_{i}+$ const, where $\varphi_{i}$ can take on a value from the discrete series $\frac{2 \pi}{N}(i-1), i=1, . ., N$.

It should be noted that, in principle, the obtained mechanism of generation of two-day photochemical oscillations cannot explain the subcritical excitation of the modes of behavior with odd periods, e.g. periods of three days, which is demonstrated by the initial photochemical system (see Fig. 3). However, it works well for all consequent period doubling bifurcations when supercritical excitation of subharmonic oscillations with periods of $6,12,24$, etc. days takes place.

It was mentioned in the Introduction that diurnal variation of solar radiation is the key mechanism of the Sun's influence on the chemical processes in the entire atmosphere of the Earth. However, but for MPCS, no other atmospheric photochemical systems are known which have similar nonlinear dynamic properties. Besides, the atmosphere is characterized by a sufficient amount of other periodic actions with large enough amplitudes (including those of the anthropogenic origin), which, in principle, can lead to the nonlinear response of the atmospheric photochemistry in other regions. Based on the derivation of the oscillator equation performed in Sect. 3, one can formulate basic preconditions of this phenomenon. First of all, the external periodic forcing should be sufficiently strong: the higher its frequency, the higher its amplitude will be. On the other hand, the excitation period should be of the order of the characteristic time of the evolution of the considered system. The presence of the catalytic cycle of destruction of some chemical component $x$ in this system is actually unnecessary. The required linear term $^{3}$ in the balance equation for this component can, e.g. ensure a chemical source proportional to the concentration of the other component, $y$. Specifically, this may occur when $x$ is born as a result of the bimolecular reaction $y+z \rightarrow x$,

\footnotetext{
3 as in the first equation of system (10).
} 
and the relative change in the third variable $z$ is small compared to the relative changes in the first two variables, i.e. $z \approx$ const.

We also formulated a simplified system of two differential nonlinear equations for analytical study of the effect of arising of reaction-diffusion waves in the MPCS. It was shown that this system has subharmonic oscillations with a period of two days and, in general, a complete spectrum of such oscillations with periods of $3,4,5$, etc. days, up to chaos. Spectral analysis verified that the harmonic component with a period of two days is best pronounced in the two-day solution of this system, while there are no harmonics with periods $1,0.5$, 0.25 , etc. days. In addition, the nonlinearity of this system has a power-law character, which, in principle, is a favorable feature for the harmonic analysis. The numerical study of this system, with diffusion taken into consideration, revealed the possibility of formation of reaction-diffusion waves possessing all the properties of waves arising in the initial system of equations for MPCS.

It should be noted that Kulikov and Feigin (2004) used the one-dimensional (in zonal direction) model of the MPCS to demonstrate that the reaction-diffusion waves are formed, generally speaking, for any period of subharmonic oscillations. It was found that for the periods of 4, 6,8 etc. days, the situation is similar to that for the oscillations with a period of two days, i.e. the waves are caused by the zonal nonuniformity of the phase of diurnal illumination variations. Their velocities are directly proportional to the diffusion coefficient $D$ and inversely proportional to the length of the zonal circle. In turn, for periods of 3, 5, 7 etc. days, the situation is basically different. First of all, nonuniformity of the phase of diurnal illumination variations is not a necessary condition for formation of such waves, which makes it possible for them to propagate in any horizontal direction rather than only along the zonal coordinate. Their velocity is proportional to $\sqrt{D}$, inversely proportional to the oscillation period and, in general, weakly depends on the length of the zonal circle. Note that, actually, the derived system of two differential nonlinear equations allows analytical study of the mechanism of reaction-diffusion waves for any type of subharmonic oscillations. Such a study performed within the framework of the theory of solitons will be presented in a separate paper.

Acknowledgements. The work was supported by the Program of Fundamental Research of the RAS Department of Physical Sciences "Physics of the Atmosphere: Electric Processes, Radiophysical Methods of Research" (project 2.1), the Russian Foundation for Basic Research (projects 10-05-01112 and 12-0500999-a), the Russian Federal Special Program "Scientific and scientifically-pedagogical staff of innovative Russia" (contract 14.740.11.1025), and the Government of the Russian Federation (contract 11.G34.31.0048).

Edited by: U. Feudel

Reviewed by: two anonymous referees

\section{References}

Ebel, A.: Eddy diffusion models for the mesosphere and lower thermosphere, J. Atmos. Terr. Phys., 42, 617-628, 1980.

Doyle, J. D.: Wind measurements in the upper atmosphere, Ph.D. thesis, Univ. of Adelaide, Adelaide, Australia, 1968.

Feigin, A. M. and Konovalov, I. B.: On the possibility of complicated dynamic behavior of atmospheric photochemical systems: Instability of the Antarctic photochemistry during the ozone hole formation, J. Geophys. Res., 101, 26023-26038, 1996.

Feigin, A. M., Konovalov, I. B., and Molkov, Ya. I.: Towards understanding nonlinear nature of atmospheric photochemistry: Essential dynamic model of the mesospheric photochemical system, J. Geophys. Res., 103, 25447-25460, 1998.

Fichtelmann, B. and Sonnemann, G.: Non-linear behavior in the photochemistry of minor constituents in the upper mesosphere, Ann. Geophys., 10, 719-728, 1992, http://www.ann-geophys.net/10/719/1992/.

Field, R. J. and Burger, M. (Eds.): Oscillations and traveling waves in chemical systems, J. Wiley and Sons, Inc., New York, 1985.

Hadac, O. and Schreiber, I.,: Stoichiometric network analysis of the photochemical processes in the mesopause region, Phys. Chem. Chem. Phys., 13, 1314-1322, 2011.

Konovalov, I. B. and Feigin, A. M.: Toward an understanding of the nonlinear nature of atmospheric photochemistry: Origin of the complicated dynamic behaviour of the mesospheric photochemical system, Nonlin. Processes Geophys., 7, 87-104, doi:10.5194/npg-7-87-2000, 2000.

Kulikov, M. Yu.: Formation of coherent spatial structures in the reactive-diffusion atmospheric system under the action of a planetary wave, Radiophys. Quantum El., 47, 662-673, 2004.

Kulikov, M. Yu.: Theoretical investigation of the influence of a quasi 2-day wave on nonlinear photochemical oscillations in the mesopause region, J. Geophys. Res., 112, D02305, doi:10.1029/2005JD006845, 2007.

Kulikov, M. Yu. and Feigin, A. M.: Reaction-Diffusion Waves in Mesospheric Photochemical System with Regard to Horizontal Turbulent Diffusion, Bulletin of the Russian Academy of Sciences: Physics, 68, 2017-2024, 2004.

Kulikov M. Yu. and Feigin, A. M.: Reactive-diffusion waves in the mesospheric photochemical system, J. Adv. Space Res., 35, 1992-1998, 2005.

Kulikov, M. Yu. and Gashturi, A. P.: The influence of vertical advection on the nonlinear dynamic properties of the upper mesosphere photochemistry, Radiophys. Quantum El., 49, 949-955, 2006.

Lubken, F. J.: Seasonal variation of turbulent energy dissipation rates at high latitudes as determined by in situ measurements of neutral density fluctuations, J. Geophys. Res., 102, 1344113456, 1997.

Muller, H. G.: Long-period meteor wind oscillations, Philos. Trans. R. Soc. London, Ser. A, 271, 585-598, 1972.

Sandford, D. J., Schwartz, M. J., and Mitchell, N. J.: The wintertime two-day wave in the polar stratosphere, mesosphere and lower thermosphere, Atmos. Chem. Phys., 8, 749-755, doi:10.5194/acp-8-749-2008, 2008.

Sonnemann, G. R.: The photochemical effects of dynamically induced variations in solar insolation, J. Atmos. Sol. Terr. Phys., 63, 781-797, 2001. 
Sonnemann, G. and Feigin, A. M: Non-linear behavior of a reaction-diffusion system of the photochemistry within the mesopause region, Phys. Rev. E, 59, 1719-1726, 1999.

Sonnemann, G. and Fichtelmann, B.: Enforced oscillations and resonances due to internal non-linear processes of photochemical system in the atmosphere, Acta. Geod. Geophys. Mont. Hung., 22, 301-311, 1987.

Sonnemann, G. and Fichtelmann, B.: Subharmonics, cascades of period of doubling and chaotic behavior of photochemistry of the mesopause region, J. Geophys. Res., 101, 1193-1203, 1997.
Sonnemann, G. R. and Grygalashvyly, M.: On the two-day oscillations and the day-to-day variability in global 3-D-modeling of the chemical system of the upper mesosphere/mesopause region, Nonlin. Processes Geophys., 12, 691-705, doi:10.5194/npg-12691-2005, 2005.

Sonnemann, G., Feigin, A. M., and Molkov, Ya. I.: On the influence of diffusion upon the nonlinear behavior of the photochemistry of the mesopause region, J. Geophys. Res., 104, 30591-30603, 1999. 